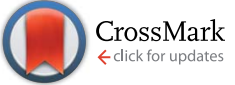

Cite this: RSC Adv., 2015, 5, 81142
Received 15th June 2015

Accepted 10th September 2015

DOI: $10.1039 / c 5 r a 11387 c$

www.rsc.org/advances

\section{Biobased Janus molecule for the facile preparation of water solutions of few layer graphene sheets}

\author{
Maurizio Galimberti, ${ }^{* a}$ Vincenzina Barbera, ${ }^{a}$ Silvia Guerra, ${ }^{a}$ Lucia Conzatti, ${ }^{b}$ \\ Chiara Castiglioni, ${ }^{a}$ Luigi Brambilla ${ }^{a}$ and Andrea Serafini ${ }^{a}$
}

\begin{abstract}
A biobased Janus molecule was used to prepare water solutions of nano-stacks made by few layer graphene. The Janus molecule was 2-(2,5-dimethyl- $1 H$-pyrrol-1-yl)-1,3-propanediol (serinol pyrrole, SP), a serinol derivative obtained through the neat reaction of 2-amino-1,3-propandiol with 2,5-hexanedione, with atom efficiency of about $85 \%$. SP contains the pyrrole ring, suitable for the interaction with carbon allotropes and hydroxy groups, that can easily interact with polar surroundings. Adducts, with about $14 \%$ by mass of SP, were prepared by reacting SP with a high surface area nano-sized graphite (HSAG), with about 35 graphene layers stacked in crystalline domains. Green methods were adopted, such as ball milling (HSAG-SP-M) and heating. Infrared spectra revealed peaks due to both HSAG and SP and additional peaks that could be attributed to the adduct. Both thermal and mechanical reactions left substantially unaltered the order in the graphitic layers and the interlayer distance, as shown by X-ray diffraction patterns. The relative intensity of the $G$ and $D$ band in the Raman spectrum was not modified by the thermal reaction, whereas enhancement of the $D$ peak was observed after ball milling. Stable water solutions of HSAG-SP-M were prepared in a concentration range from 0.1 to $1 \mathrm{mg} \mathrm{mL}^{-1}$. Centrifugation allowed isolation of adducts with few stacked graphene layers, as revealed by high resolution transmission electron microscopy. An image of the adduct showed an organic layer tightly adhered to the carbon surface. SP appears a suitable molecule for the easy functionalization of carbon allotropes, such as nanostacks of graphene layers, without substantially affecting the bulk crystalline organization and promoting the separation of the aggregates into stacks containing a low/very low number of graphene layers.
\end{abstract}

\section{Introduction}

Since the discovery of fullerene, ${ }^{1}$ nano-sized carbon allotropes have been the subject of huge amounts of research activity. Carbon nanotubes (CNTs), both single ${ }^{2,3}$ and multi-walled, ${ }^{\mathbf{4 , 5}}$ graphene $(\mathrm{G}),{ }^{6-9}$ or graphitic nanofillers made by few layers of graphene $\mathrm{e}^{\mathbf{1 0 - 1 3}}$ are studied as they dramatically improve properties such as electrical and thermal conductivity, mechanical reinforcement, thermal and flame resistance, of both thermoplastic and elastomeric matrices. ${ }^{14}$ In recent years, attention has been in particular focussed on graphene, in consideration of its huge surface area, exceptional mechanical strength and high carrier mobility. ${ }^{\mathbf{6 - 9 , 1 5 - 1 8}}$

Single or few layers of graphene can be obtained through micromechanical cleavage, ${ }^{6,19}$ epitaxial growth and chemical vapour deposition of graphene films, ${ }^{20-27}$ liquid phase exfoliation. ${ }^{28-33}$ Much interest has been directed, particularly in view of large scale production, to the oxidation of graphite ${ }^{34-38}$ to graphite

${ }^{a}$ Politecnico di Milano, Department of Chemistry, Materials and Chemical Engineering "G. Natta”, Via Mancinelli 7, 20131 Milano, Italy. E-mail: maurizio.galimberti@ polimi.it

${ }^{b}$ National Council of Research, Institute for the Study of Macromolecules, Via De Marini 6, 16149 Genova, Italy oxide $(\mathrm{GO})^{39-44}$ followed by thermal or chemical reduction. ${ }^{45-49}$ Such method gives also the chance of preparing GO. The precise structure of GO is still unknown. ${ }^{41}$ However, the adopted oxidation methods lead to extensive modification of the graphene layers. It was reported ${ }^{40}$ that hydroxy and epoxide groups are mainly present on the surface of basal planes and carbonyl and carboxyl groups are bounded to the edges. GO can be dispersed in polar media and can be the platform for further functionalizations..$^{50-62}$ The oxidation-reduction method is however characterized by several drawbacks. The oxidation requires strong acids, explosive oxidation agents, harsh reaction conditions and long reaction times. ${ }^{35-38}$ Thermal reduction occurs at high temperature and under controlled conditions, efficient chemical reducing agents could be toxic, hazardous and expensive, such as, for instance, those based on hydrazine.$^{63,64}$ Besides that, it is increasingly acknowledged that the complete restoration of $\mathrm{sp}^{2}$ hybridization of graphene carbons can be hardly achieved.

Aim of this work was the introduction, on graphene layers, of oxygen containing functional groups, in particular of hydroxy groups, without substantially affecting the $\mathrm{sp}^{2}$ hybridization of the carbon atoms, thus keeping structural order inside graphene sheets. The achievement of this goal could allow the easy preparation of solutions in polar media (e.g. in water) of 


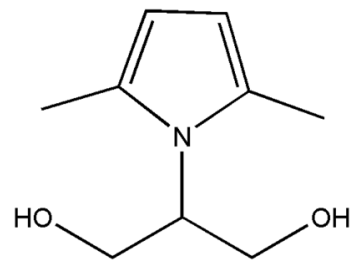

Fig. 1 2-(2,5-Dimethyl-1H-pyrrol-1-yl)-1,3-propanediol (SP).

graphene layers with almost unchanged thermal and electrical conductivity, without the need of further chemical treatments. The peculiar objective of the work was to perform the modification of graphene layers through simple reactions inspired to the principles of green chemistry, ideally by using biobased chemicals.

To pursue these objectives, 2-amino-1,3-propanediol, known as serinol (S), was identified as the starting building block. Serinol can be directly obtained from renewable sources ${ }^{65}$ and is commercially available, arising from the reaction of easily available chemicals such as glycerol. The chemoselectivity of its amino and hydroxyl groups was exploited ${ }^{66,67}$ to perform PaalKnorr reaction ${ }^{\mathbf{6 8 , 6 9}}$ of $\mathbf{S}$ with 2,5-hexanedione (HD) obtaining 2-(2,5-dimethyl-1H-pyrrol-1-yl)-1,3-propanediol, shown in Fig. 1 and hereinafter indicated as serinol pyrrole (SP).

The reaction was performed in the absence of solvents and catalysts, with yield of about $95 \%$ and atomic efficiency of about $85 \%$. SP contains two hydroxyl groups, that promote the compatibility with polar surroundings such as water, eco-friendly solvents as alcohols, ethers and esters and polar polymer matrices. SP contains as well as pyrrole ring, that could favour $\pi-$ $\pi$ interaction with the aromatic rings of carbon allotropes. SP appears as a Janus Bifrons molecule, suitable for the preparation of solutions of graphitic nanofillers in polar media. ${ }^{66,67,70,71}$

Reaction was performed between SP and a nano-sized graphite with very high surface area (HSAG), higher than $300 \mathrm{~m}^{2} \mathrm{~g}^{-1}$, commercially available and obtained through milling. HSAG was characterized by a relatively low number of layers stacked in the direction orthogonal to graphene planes (about 30) and by a high shape anisotropy, defined as the ratio between the crystallites size in directions orthogonal and parallel to the layers. ${ }^{72}$ The interaction of SP and HSAG was promoted by mechanical and thermal energy. Adducts were studied by means of elemental, thermogravimetric (TGA), infrared (IR), wide angle X-ray diffraction (WAXD), transmission electron microscopy (TEM) analysis and Raman spectroscopy. Dispersions of HSAG-SP adducts in water were studied through dynamic light scattering (DLS) and UV spectroscopy. HSAG-SP adducts isolated through centrifugation of supernatant solutions were analyzed through high resolution TEM (HR-TEM). Electrical conductivity of HSAG-SP adducts was investigated on sheets deposited on paper.

\section{Experimental}

\subsection{Materials}

Reagents and solvents commercially available were purchased and used without further purification: 2,5-hexandione (MerckSchuchardt), 2-amino-1,3-propanediol (kindly provided by Bracco), acetone (Aldrich), high surface area graphite (HSAG) with Synthetic Graphite $8427 \circledR$ as trademark was purchased from Asbury Graphite Mills Inc., with a minimum carbon mass \% of 99.5 , a surface area of $330 \mathrm{~m}^{2} \mathrm{~g}^{-1}$, the number of stacked layers in the orthogonal direction to graphitic planes is about $35 .^{72}$

\subsection{Synthesis of 2-(2,5-dimethyl-1H-pyrrol-1-yl)-1,3- propanediol (SP)}

2-(2,5-Dimethyl-1H-pyrrol-1-yl)-1,3-propanediol was prepared following the reaction scheme shown in Scheme $1 .^{67}$

A mixture of hexan-2,5-dione (41.4 g; $0.36 \mathrm{~mol}$ ) and serinol ( $30.0 \mathrm{~g} ; 0.33 \mathrm{~mol}$ ) was poured into a $100 \mathrm{~mL}$ round bottomed flask equipped with magnetic stirrer. The mixture was then stirred, at room temperature, for $6 \mathrm{~h}$. The resulting compound 4a,6a-dimethyl-hexahydro-1,4-dioxa- $6 b$-azacyclopenta[ $c d]$ pentalene (HHP) was characterized through ${ }^{1} \mathrm{H}$ NMR and the yield was estimated to be $99 \% .{ }^{1} \mathrm{H}$ NMR (400 MHz, DMSO- $d 6, \delta$ in ppm): 1.28 (s, 6H); 1.77 (m, 2H); $1.93(\mathrm{~m}, 2 \mathrm{H}) ; 3.60(\mathrm{~m}, 4 \mathrm{H})$; $3.94(\mathrm{q}, 1 \mathrm{H}) .{ }^{67,68}$

The product mixture obtained from the synthesis of HHP was kept under vacuum for $2 \mathrm{~h}$ and then heated to $180{ }^{\circ} \mathrm{C}$ for $50 \mathrm{~min}$. After distillation under reduced pressure at $130^{\circ} \mathrm{C}$ and 0.1 mbar, SP was isolated as yellow oil with $96 \%$ yield (see Fig. 1 and Scheme 1). The global yield of the two step synthesis was therefore about 95\%. ${ }^{1} \mathrm{H}$ NMR (400 MHz, DMSO- $d 6, \delta$ in ppm): $2.16\left(\mathrm{~s}, 6 \mathrm{H},-\mathrm{CH}_{3}\right.$ at $\mathrm{C}-2,5$ of pyrrole moiety); $3.63(\mathrm{~m}, 2 \mathrm{H}$, $\left.\mathrm{CH}_{2} \mathrm{OH}\right) ; 3.76\left(\mathrm{~m}, 2 \mathrm{H}, \mathrm{CH}_{2} \mathrm{OH}\right) ; 4.10$ (quintet, $1 \mathrm{H}$, at $\mathrm{C}-3$ of diol); $4.73\left(\mathrm{t}, 2 \mathrm{H}, \mathrm{CH}_{2} \mathrm{OH}\right) ; 5.55$ (s, 2H, C-3,4 of pyrrole moiety). ${ }^{67,68}$

\subsection{Synthesis of HSAG-SP adducts}

HSAG-SP adducts were prepared by reacting HSAG and SP with the help of either mechanical (HSAG-SP-M) or thermal (HSAG-SP-T) energy.

2.3.1. Synthesis of HSAG-SP-M adduct. In a $100 \mathrm{~mL}$ round bottomed flask was put in sequence graphite $(5 \mathrm{~g}, 66 \mathrm{mmol})$ and acetone $(15 \mathrm{~mL})$. The suspension was sonicated for $15 \mathrm{~min}$, using
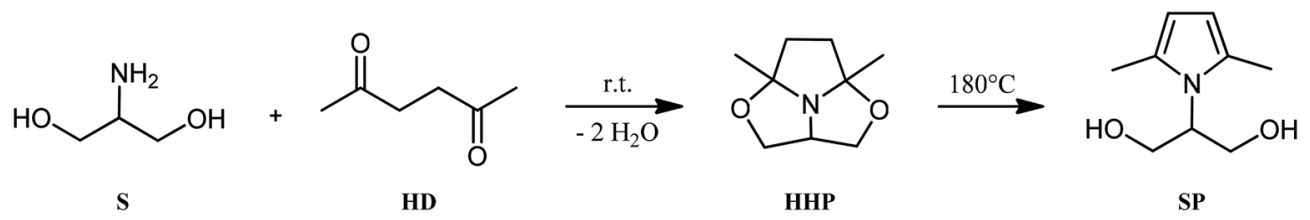

Scheme 1 Reaction pathway for the preparation of the serinol derivatives HHP and SP. 
a $2 \mathrm{~L}$ ultrasonic bath. After this time, a solution of SP (5.87 g, $66 \mathrm{mmol}$ ) in acetone $(15 \mathrm{~mL})$ was added. The resulting suspension was sonicated for $15 \mathrm{~min}$. The solvent was removed under reduced pressure. The black powder of graphite/SP was treated using a planetary ball mill S100 from Retsch, having the grinding jar moving in a horizontal plane, with a volume of $0.3 \mathrm{~L}$. The grinding jar was loaded with 6 ceramic balls having a diameter of $20 \mathrm{~mm} .10 .87 \mathrm{~g}$ of graphite/SP adduct were put into the jar, that was allowed to rotate at $300 \mathrm{rpm}$, at room temperature, for $6 \mathrm{~h}$. After this time, the mixture was placed in a Büchner funnel with a sintered glass disc. It was repeatedly washed with distilled water $(6 \times 100 \mathrm{~mL})$ obtaining $9.8 \mathrm{~g}$ of black powder. ${ }^{71}$

2.3.2. Synthesis of HSAG-SP-T adduct. In a $100 \mathrm{~mL}$ round bottom flask was put in sequence graphite $(5 \mathrm{~g}, 66 \mathrm{mmol})$ and acetone $(15 \mathrm{~mL})$. The suspension was sonicated for $15 \mathrm{~min}$, using a $2 \mathrm{~L}$ ultrasonic bath. After this time, a solution of SP $(1.116 \mathrm{~g}, 6.6 \mathrm{mmol})$ in acetone $(5 \mathrm{~mL})$ was added. The resulting suspension was sonicated for $15 \mathrm{~min}$. The solvent was removed under reduced pressure. The black powder of graphite/SP $(6.10 \mathrm{~g})$ was poured into a $100 \mathrm{~mL}$ round bottomed flask equipped with magnetic stirrer and was heated at $130{ }^{\circ} \mathrm{C}$ for $6 \mathrm{~h}$. After this time, the mixture was placed in a Büchner funnel with a sintered glass disc. It was repeatedly washed with distilled water $(3 \times 20 \mathrm{~mL})$, obtaining $5.86 \mathrm{~g}$ of black powder. ${ }^{71}$

\subsection{Characterization of HSAG-SP adducts}

2.4.1. Thermogravimetric analysis. TGA tests under flowing $\mathrm{N}_{2}\left(60 \mathrm{~mL} \min ^{-1}\right)$ were performed with a Mettler TGA SDTA/851 instrument according to the standard method ISO9924-1. Samples $(10 \mathrm{mg})$ were heated from 30 to $300{ }^{\circ} \mathrm{C}$ at $10{ }^{\circ} \mathrm{C}$ $\mathrm{min}^{-1}$, kept at $300{ }^{\circ} \mathrm{C}$ for $10 \mathrm{~min}$, and then heated up to $550{ }^{\circ} \mathrm{C}$ at $20{ }^{\circ} \mathrm{C} \mathrm{min}{ }^{-1}$. After being maintained at $550{ }^{\circ} \mathrm{C}$ for $15 \mathrm{~min}$, they were further heated up to $900{ }^{\circ} \mathrm{C}$ and kept at $900{ }^{\circ} \mathrm{C}$ for 30 min under flowing air $\left(60 \mathrm{~mL} \mathrm{~min}^{-1}\right)$.

2.4.2. Elemental analysis. Elemental analysis was performed with a Thermo FlashEA 1112 Series CHNS-O analyzer, after pretreating samples in an oven at $100{ }^{\circ} \mathrm{C}$ for $12 \mathrm{~h}$.

2.4.3. FT-IR. FT-IR spectra were recorded between 450 and $4000 \mathrm{~cm}^{-1}$ by using a Perkin-Elmer FT-IR Spectrum One equipped with Universal ATR Sampling Accessory with diamond crystal.

2.4.4. Raman spectroscopy. Raman spectra of powder samples deposited on a glass slide were recorded by using a Horiba Jobin Yvon Labram HR800 dispersive Raman spectrometer equipped with Olympus BX41 microscope and a 50× objective. The excitation line at $632.8 \mathrm{~nm}$ of a He/Ne laser was kept at $0.5 \mathrm{~mW}$ in order to prevent possible samples degradation. The spectra were obtained as the average of four acquisitions (scan time: $30 \mathrm{~s}$ for each acquisition) with a spectral resolution of $2 \mathrm{~cm}^{-1}$.

2.4.5. Wide angle X-ray diffraction. Wide-angle X-ray diffraction (WAXD) patterns were obtained in reflection, with an automatic Bruker D8 Advance diffractometer, with nickel filtered $\mathrm{Cu}-\mathrm{K} \alpha$ radiation. Patterns were recorded in $10-100^{\circ}$ as the $2 \theta$ range, being $2 \theta$ the peak diffraction angle. Distance between crystallographic planes was calculated from the Bragg law. The $D_{h k \ell}$ correlation length, in the direction perpendicular to the $h k \ell$ crystal graphitic planes, was determined applying the Scherrer equation.

$$
D_{h k \ell}=K \lambda /\left(\beta_{h k \ell} \cos \theta_{h k \ell}\right)
$$

where: $K$ is the Scherrer constant, $\lambda$ is the wavelength of the irradiating beam (1.5419 $\AA, \mathrm{Cu}-\mathrm{K} \alpha), \beta_{h k \ell}$ is the width at half height, and $\theta_{h k \ell}$ is the diffraction angle. The instrumental broadening, $b$, was determined by obtaining a WAXD pattern of a standard silicon powder 325 mesh (99\%), under the same experimental conditions. The width at half height, $\beta_{h k \ell}=\left(B_{h k \ell}-\right.$ $b$ ) was corrected, for each observed reflection with $\beta_{h k \ell}<1^{\circ}$, by subtracting the instrumental broadening of the closest silicon reflection from the experimental width at half height, $B_{h k \ell}$.

\subsection{Water solutions of HSAG-SP adducts}

2.5.1. Preparation. Water solutions of HSAG-SP adducts at different concentrations $\left(1 \mathrm{mg} \mathrm{mL}{ }^{-1} ; 0.5 \mathrm{mg} \mathrm{mL}{ }^{-1} ; 0.3\right.$ $\mathrm{mg} \mathrm{mL} \mathrm{mL}^{-1} ; 0.1 \mathrm{mg} \mathrm{mL}{ }^{-1}$ ) were prepared. Each solution was sonicated for $10 \mathrm{~min}$ using an ultrasonic bath $(260 \mathrm{~W})$ and subsequently UV-vis absorption was measured. The solution (10 $\mathrm{mL}$ ) of each sample was put in a Falcon ${ }^{\mathrm{TM}} 15 \mathrm{~mL}$ Conical Centrifuge Tubes and centrifuged at: $2000 \mathrm{rpm}$ for 10 minutes and at $9000 \mathrm{rpm}$ for 5, 30, 60 and 90 minutes. Supernatants obtained after each centrifugation processes were removed and analyzed. UV-vis absorptions and DLS analysis were measured immediately after each centrifugation and after 1 week stored.

2.5.2. UV-vis spectroscopy. The suspensions of adduct (3 $\mathrm{mL}$ ) were placed by pipette Pasteur, in quartz cuvettes of $1 \mathrm{~cm}$ optical path (volume 1 or $3 \mathrm{~mL}$ ) and analyzed by a spectrophotometer Hawlett Packard 8452A Diode Array Spectrophotometer. Resets the instrument with pure solvent and has one UV spectrum from 200-340 $\mathrm{nm}$. It was recorded a white the solvent employed. The UV-visible spectrum reported intensity the absorption as a function of the wavelength of the radiation between 200 and $750 \mathrm{~nm}$.

2.5.3. Dynamic light scattering (DLS). The nanoparticle size in HSAG-SP solutions was analyzed with a Zetasizer Dynamic Light Scattering system (Malvern Instrument Ltd) at room temperature using a $1 \quad 1 / 4 \quad 632.8 \quad \mathrm{~nm}$ He-Ne laser. Each centrifuged solution, prepared using a concentration of $1 \mathrm{mg} \mathrm{mL}{ }^{-1}$, was analyzed by DLS: $3 \mathrm{~mL}$ of solution were placed using a pipette Pasteur, in a quartz cuvettes of $1 \mathrm{~cm}$ optical path and analyzed immediately after centrifugation and after 10, 60 and $100 \mathrm{~min}$ of storage.

2.5.4. Transmission electron microscopy. Water solutions (1 $\mathrm{mg} \mathrm{mL} \mathrm{mL}^{-1}$ ) of adducts were centrifuged using an ALC-Centrifugette 4206. TEM analysis of supernatants was performed with a Zeiss EM900 microscope operating at an accelerating voltage of $80 \mathrm{kV}$. Few drops of acetone diluted suspension of the sample were deposited on a carbon film supported on a standard $\mathrm{Cu}$ grid and air-dried for several hours before analysis.

2.5.5. High-resolution transmission electron microscopy (HR-TEM). HR-TEM investigations on adducts' samples, isolated from the supernatant solutions after centrifugation, were carried out with a Philips CM 200 field emission gun 
microscope operating at an accelerating voltage of $200 \mathrm{kV}$. Few drops of the water solutions were deposited on 200 mesh lacey carbon-coated copper grid and air-dried for several hours before analysis. During acquisition of HR-TEM images, the samples did not undergo structural transformation. Low beam current densities and short acquisition times were adopted. To estimate the number of stacked graphene layers and the dimensions of the stacks visible in HR-TEM micrographs, the Gatan Digital Micrograph software was used.

\section{Results and discussion}

The synthesis of SP, summarized in Scheme 1, is described in detail in the experimental part and has been discussed elsewhere. ${ }^{6,67}$

It is worth underlining the high atomeconomy of the reaction $(82 \%)$, with water as the only co-product of the first step. The high yield, about $95 \%$, was easily reproduced in all the preparations performed in this research. This led to the atomic efficiency of about $78 \%$ for the preparation of the SP sample used in this work.

The interaction of HSAG with SP was promoted with the help of mechanical or thermal energy, as described in the experimental part. The adopted procedures are summarized in the block diagram of Fig. 2 .

In brief, HSAG and SP were first premixed. HSAG-SP mixture was either ball milled in a jar for $6 \mathrm{~h}$ or heated in a flask for 6 hours at $130{ }^{\circ} \mathrm{C}$. The products taken either from the jar or from the flask were washed several times with water, until SP was undetectable in the washing water. The HSAG/SP molar amount (in the case of HSAG, the moles of aromatic rings were considered) was explored in the range from $2: 1$ to $10: 1$. Adducts prepared by using either mechanical or thermal energy were named HSAG-SP-M and HSAG-SP-T, respectively. In the present work, were characterized HSAG-SP-M and HSAG-SP-T samples having almost the same amount of SP, determined from thermogravimetric and elemental analysis.

TGA analysis was performed under nitrogen on HSAG, SP and on the HSAG-SP adducts. Thermographs are shown in Fig. 3. In Table 1, are shown data of mass loss for HSAG and for the adducts.

SP achieves its boiling point at about $230^{\circ} \mathrm{C}$. Curve of HSAG shows decomposition profile made by two main steps, that can be attributed to: decomposition of oxygen-containing and alkenylic groups for $T$ in the range from $150{ }^{\circ} \mathrm{C}$ to $700{ }^{\circ} \mathrm{C}$ and combustion with oxygen (see experimental part) at $T>700{ }^{\circ} \mathrm{C}$.

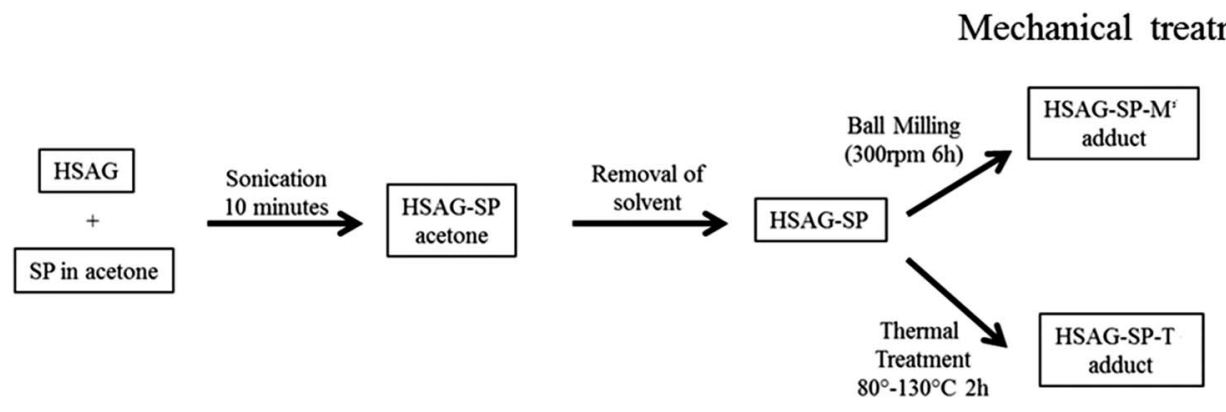

Thermal treatment

Fig. 2 Block diagram for the preparation of HSAG-SP adducts: with the help of mechanical (HSAG-SP-M) or thermal energy (HSAG-SP-T).

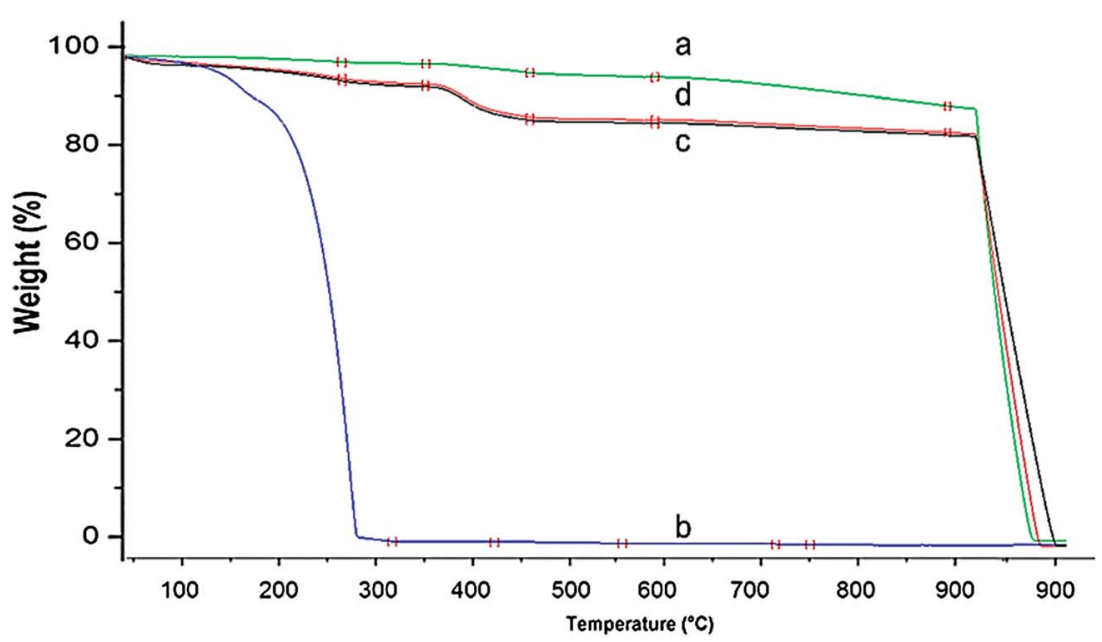

Fig. 3 TGA curves of HSAG (a), SP (b), HSAG-SP-T (c), heated HSAG-SP-M (d). 
Table 1 Mass loss for HSAG, HSAG-SP-M and HSAG-SP-T, from TGA analysis $^{a}$

\begin{tabular}{llll}
\hline & \multicolumn{3}{l}{ Mass loss [\%] } \\
\cline { 2 - 4 } Sample & $T<150{ }^{\circ} \mathrm{C}$ & $\begin{array}{l}150{ }^{\circ} \mathrm{C} \\
<T<300{ }^{\circ} \mathrm{C}\end{array}$ & $\begin{array}{l}300{ }^{\circ} \mathrm{C} \\
<T<500{ }^{\circ} \mathrm{C}\end{array}$ \\
\hline HSAG & 0 & 0 & 3.1 \\
HSAG-SP-M & 0.8 & 2.7 & 7.8 \\
HSAG-SP-T & 0.8 & 2.7 & 7.7
\end{tabular}

${ }^{a}$ Residual mass loss was above $700{ }^{\circ} \mathrm{C}$. Total mass loss was $100 \%$.

Mass loss due to water removal, that could be expected at $T<$ $150{ }^{\circ} \mathrm{C}$, can not be detected.

Larger mass loss in the temperature range from 150 to $500{ }^{\circ} \mathrm{C}$ is shown by HSAG-SP-T and HSAG-SP-M. Degradation occurs at a temperature much higher than the one observed in the SP curve, indicating that residual SP is not present on HSAG. Both the adducts reveal an appreciable mass loss at temperatures below $150{ }^{\circ} \mathrm{C}$. Interestingly, the larger amount of mass loss at $T>150{ }^{\circ} \mathrm{C}$ is accompanied by larger mass loss at $T<150{ }^{\circ} \mathrm{C}$. It could be hypothesized that HSAG-SP adducts that contain larger amount of oxygenated functional groups hold larger amount of water. The onset temperature of the last step degradation is pretty similar for HSAG and for the adducts, indicating that the reaction with SP did not lead to lower thermal stability of HSAG.

As reported in the Experimental part, pristine HSAG had 99.5\% as carbon content. The nitrogen content of HSAG-SP adducts was determined by means of elemental analysis. Table 2 shows the experimental values and the amount of SP, theoretically estimated.

The adducts contain comparable amount of SP, $13 \%$ and $14 \%$ by mass. HSAG-SP-T was obtained by adopting $10 / 1$ as the HSAG/SP molar ratio, whereas $2 / 1$ as molar ratio was used for the preparation of HSAG-SP-M. Studies have not been performed on the yield of the reaction between HSAG and SP as a function of the preparation procedure. As mentioned above, samples discussed in this work were selected as they show comparable amount of SP.

Functional groups present on HSAG-SP adducts were investigated by means of IR spectroscopy. Fig. 4 shows the IR spectra of pristine HSAG (a), SP (b) and of HSAG/SP mixture (HSAG/SP $=2 / 1$ as molar ratio) (c). Such mixture was prepared by simply mixing HSAG and SP, without giving either thermal or

Table 2 Mass\% of nitrogen and of SP

\begin{tabular}{lcc}
\hline & \multicolumn{2}{l}{ Adduct } \\
\cline { 2 - 3 } Mass\% of & HSAG-SP-T & HSAG-SP-M \\
\hline Nitrogen $^{a}$ & 1.96 & 1.97 \\
SP $^{b}$ & 14.03 & 13.00
\end{tabular}

${ }^{a}$ From elemental analysis. ${ }^{b}$ Calculated on the basis of the nitrogen content.

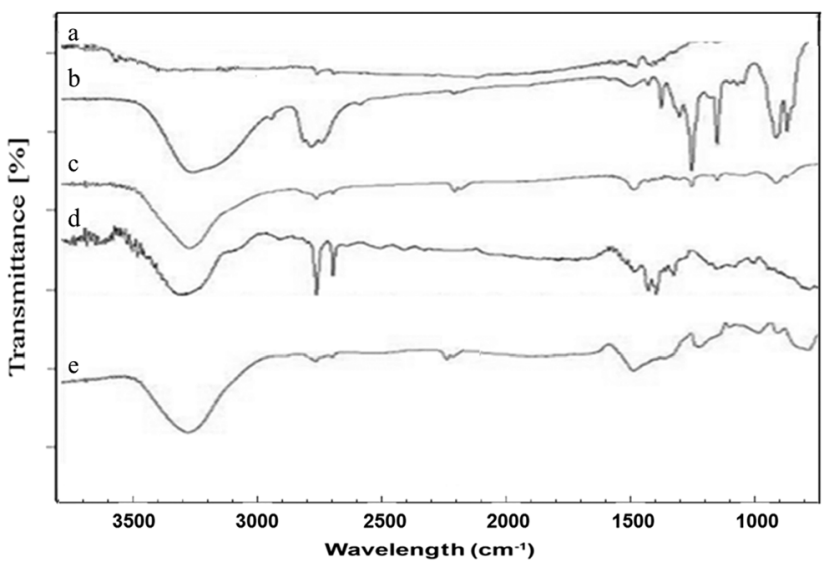

Fig. 4 IR spectra of HSAG (a), SP (b), HSAG/SP = 2/1 (c), HSAG-SP-M adduct (d), heated HSAG-SP-T adduct (e).

mechanical energy. Spectra of HSAG-SP-T and HSAG-SP-M adducts are in Fig. 4d and in Fig. 4e, respectively.

In the SP spectrum (Fig. 4b), band in the region from $3370 \mathrm{~cm}^{-1}$ to $3330 \mathrm{~cm}^{-1}$ can be reasonably attributed to hydrogen bonded $\mathrm{OH}$ groups, the pyrrole ring is evidenced by the collective vibration mode of $\mathrm{C}=\mathrm{C} / \mathrm{C}-\mathrm{C}$ stretchings, located at about $1530 \mathrm{~cm}^{-1}$ and by other bands at 1395 and $1490 \mathrm{~cm}^{-1}$, related to vibrational modes involving the pyrrole unit. The above bands due to pyrrole ring can be taken as fingerprint of SP. These bands are still clearly detectable in the spectrum of the mixture (Fig. 4c) where they do not show frequency shifts with respect to pristine SP (Fig. 4b). Spectra of both washed adducts (Fig. $4 \mathrm{~d}$ and e) show bands that cannot be attributed to HSAG. In particular, they are: bands in the region of $\mathrm{sp}^{3} \mathrm{CH}$ stretching at about $2900 \mathrm{~cm}^{-1}$ and features in the region characteristic of the $\mathrm{C}-\mathrm{C}$ stretchings of aromatic rings, namely the bands at 1590 and $1470 \mathrm{~cm}^{-1}$, peaks at $1383 \mathrm{~cm}^{-1}$ and $956 \mathrm{~cm}^{-1}$ which could be attributable to vibrations involving the diole function and the alkenyl groups of reacted SP molecules. These findings confirm what already observed through elemental and TGA analysis, that means the formation of a stable HSAG-SP adduct. Moreover, they also seem to suggest the occurring of a reaction or at least of an interaction able to modify the nature and/or the surrounding of the chemical bonds in the pyrrole ring of SP. This aspect is under investigation. As working hypothesis, Diels-Alder reaction between dangling double bonds of graphene layers and the pyrrole rings is taken into consideration.

Organization at the solid state of HSAG and of HSAG-SP adducts was investigated by means of WAXD. Fig. 5 shows WAXD patterns of HSAG (a), HSAG-SP-M (b), HSAG-SP-T (c).

Assignment of reflections due to pristine HSAG is shown in Fig. 5a. Patterns of HSAG-SP-T and HSAG-SP-M reveal that the (002) reflection remains at the same $2 \theta$ value as in the pattern of pristine HSAG. This finding indicates that $\mathbf{S P}$ was not intercalated in the interlayer space. The number of stacked layers was calculated by applying the Scherrer equation (see experimental section) to the (002) reflection. Such number was found to be: 35 for HSAG, 24 for HSAG-SP-M, and 29 for HSAG-SP-T. It 


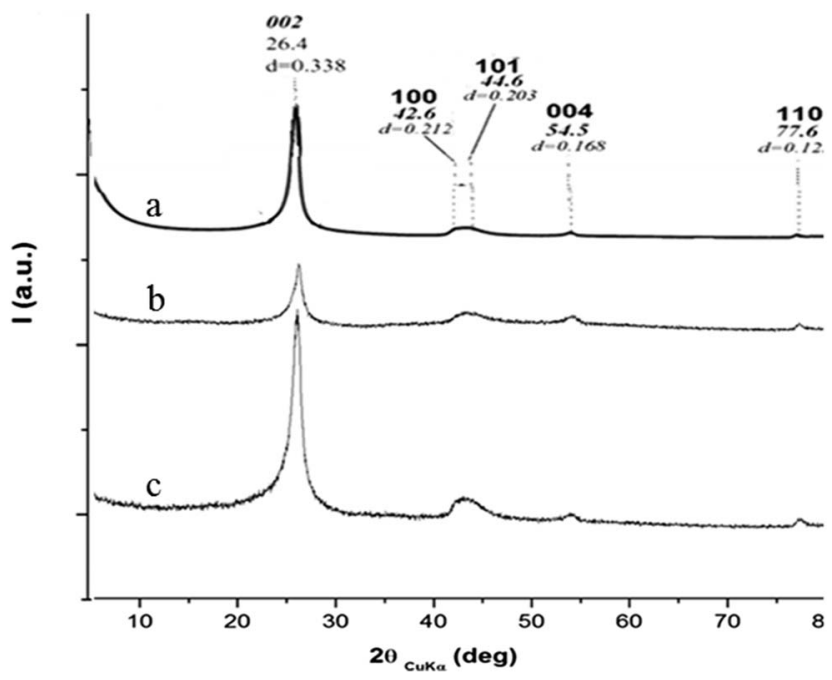

Fig. 5 WAXD patterns of HSAG (a), milled HSAG-SP-M (b), HSAG-SP-T (c).

appears that the functionalization reaction promotes the reduction of the crystalline order in the direction orthogonal to the graphene layers. 100 and 110 reflections indicate the in plane correlation lengths. The in plane order was estimated by applying the Scherrer equation to the 110 reflection: the correlation length was found, for HSAG and for the adducts, in a range from $26.5 \mathrm{~nm}$ to $28 \mathrm{~nm}$. This result seems to suggest that the reaction of HSAG with SP, also via ball milling, does not substantially alter the in plane order.

Structural characterization of HSAG and HSAG-SP adducts was performed via Raman spectroscopy, a tool widely employed for the study of carbonaceous materials. ${ }^{73-81}$ In Raman spectrum, two lines, named D and $G$ and located at $1350 \mathrm{~cm}^{-1}$ and $1590 \mathrm{~cm}^{-1}$ respectively, reveal the presence of graphitic $\mathrm{sp}^{2}$-phase. In particular, the $\mathrm{G}$ peak is characteristic of bulk crystalline graphite (graphene), whereas the $\mathrm{D}$ peak appears in the presence of either disorder or confinement (e.g. by edges) of the graphitic layers. ${ }^{77-82}$ Structural defects can be holes, $\mathrm{sp}^{3}$ or sp carbon atoms, free radicals, distortions from planarity, grafted functional groups. Moreover, it is worth reminding that graphitic layers have finite dimensions and irregular boundaries with, for example, dangling bonds. All these defects give rise to Raman scattering in the $\mathrm{D}$ region with a frequency that depends on the excitation wavelength. ${ }^{83}$ Some of the authors performed ball milling of graphitic powders, ${ }^{\mathbf{8 4}}$ in order to vary the average crystallite size and the amount of defects. Raman spectrum showed evident D line, whose intensity increased with the grinding time. Fig. 6 shows Raman spectra of HSAG (Fig. 6a) HSAG-SP-T (Fig. 6b) and HSAG-SP-M (Fig. 6c). Analysis was performed on a good number of spots and spectra can be considered representative of the investigated samples.

In all the spectra, $\mathrm{G}$ and $\mathrm{D}$ bands are present, with similar intensity. Interestingly, larger intensity can be observed for the $\mathrm{D}$ band in the spectrum of the adduct prepared through ball milling, whereas relative intensity of $\mathrm{G}$ and $\mathrm{D}$ peak appears substantially unaltered after the thermal treatment of HSAG

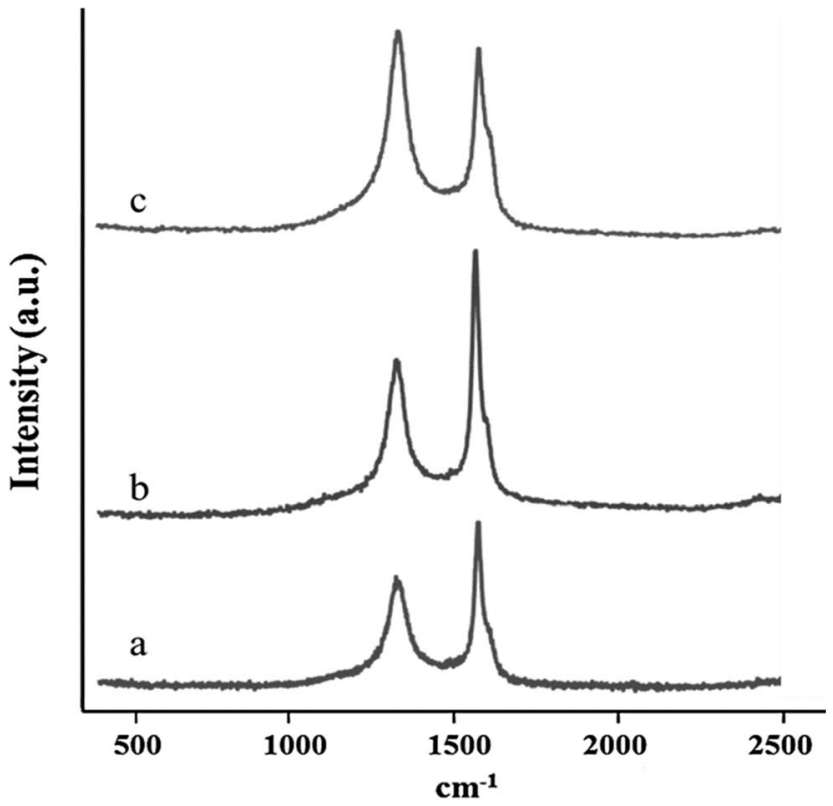

Fig. 6 Raman spectra of HSAG (a) HSAG-SP-T (b), HSAG-SP-M (c).

with SP. Hence, there are not indications that the reaction of HSAG with SP appreciably alter per se the structure of the graphitic layers, whereas the milling process appears to play the major role. The interpretation proposed by some of the authors to comment Raman spectra of ball milled graphite ${ }^{\mathbf{8 4}}$ can be applied also in the present work. Graphitic samples have a core sufficiently far from the edge, with electronic and vibrational properties that can not be distinguished from those of infinite and ideal graphene layers. A confined crown region close to the edge is electronically perturbed and local electronic and vibrational structures, affected by confinement effects, are different from those of the bulk materials. At the very edges, many types of molecular disorder are possible, making the system different from the ideal $\mathrm{sp}^{2}$ carbon layer. In consideration of findings from WAXD analysis, the enhancement of the D band in the Raman spectrum of HSAG-SP-M could be prevailingly attributed, at least as working hypothesis, to larger amounts of defects in the crown region and at the edges.

On the basis of the results discussed so far and in the light of what available in the scientific literature ${ }^{84}$ it seems possible to comment that HSAG-SP adducts are nano-stacks of confined graphene, with peripheral functional groups containing oxygen atoms, in particular hydroxyl groups.

As it should be expected, considering the presence of such functional groups, water dispersions of HSAG-SP were easily prepared (as described in the experimental part). Such dispersions were analyzed by UV-vis absorption. Dispersions freshly prepared and after $30 \mathrm{~min}$ centrifugation at $2000 \mathrm{rpm}$ of HSAG-SP-M adducts are shown in Fig. 7 (Fig. 7b and c, respectively), together with a suspension of pristine HSAG (Fig. 7a). Decantation can not be observed for the dispersions based on HSAG-SP adducts.

UV-vis absorption analysis was performed on water dispersions of HSAG-SP-M adduct with $0.1,0.3,0.5$ and 1 

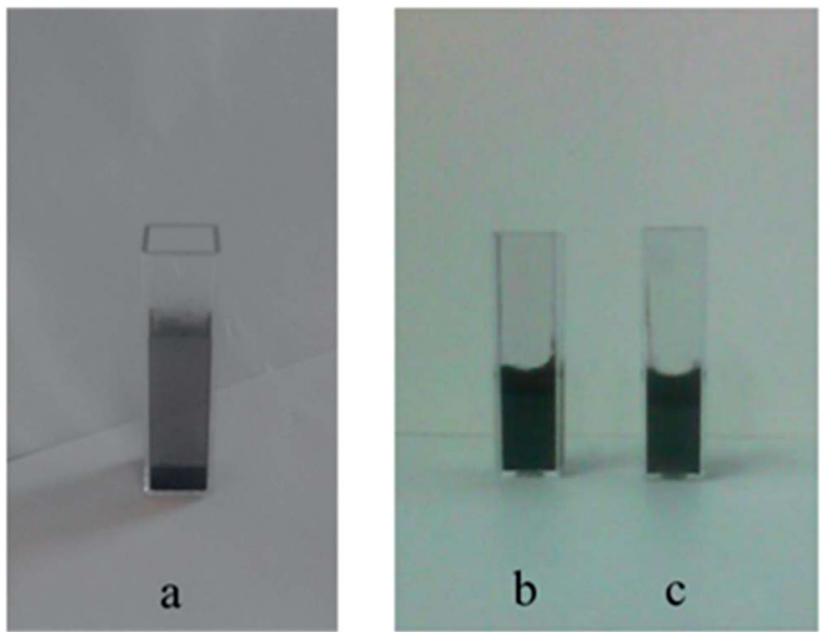

Fig. 7 Water dispersions of HSAG (a), HSAG-SP-M: freshly prepared (b), after 30 min centrifugation at $2000 \mathrm{rpm}$ (c).

$\mathrm{mg} \mathrm{mL} \mathrm{m}^{-1}$ as adduct concentration. In Fig. $8 \mathrm{a}$ it is shown that the absorbance monotonously increases with the adduct concentration. Plot of UV-vis absorbance as a function of the adduct concentration (not reported in this manuscript) reveals a linear correlation, suggesting that water solutions of HSAG-SP-M were prepared. The stability of the solution with $1 \mathrm{mg} \mathrm{mL} \mathrm{m}^{-1}$ as the adduct concentration was investigated by taking UV-vis spectra on a samples stored for 1 week and on samples centrifuged at $9000 \mathrm{rpm}$ for 30 minutes and 1 hour respectively. In Fig. $8 \mathrm{~b}$, it is evident that the same absorbance was measured for the suspensions freshly prepared and stored for 1 week, confirming that the adduct did not settle down, even after long time. Moreover, it is possible to observe that only the centrifugation at $9000 \mathrm{rpm}$ led to appreciable reduction of absorbance, that nonetheless remains at a remarkable level.

Average size of particles of HSAG and HSAG-SP adducts, in water dispersions, was determined by means of DLS (as reported in the experimental part). Table 3 shows data for as prepared dispersions and for the supernatant dispersions after $30 \mathrm{~min}$ centrifugation at $9000 \mathrm{rpm}$.
Values obtained by DLS measurements represent the hydrodynamic radius of HSAG particles in the dispersions. It appears that particles of HSAG-SP-M have lower average size than particles of HSAG. The average size further decreases in the supernatant suspension. On the contrary, the average size appears to increase, with respect to HSAG, when the HSAG-SP adduct is prepared by heating. Also the average size in the supernatant suspension is larger than in HSAG. It seems thus that the functional groups can favour the aggregation of HSAG particles that experience thermal treatment. The average size of HSAG-SP-M aggregates in solution was investigated at different times ( $t=0,10,60,100 \mathrm{~min})$, after centrifugation at $9000 \mathrm{rpm}$ for $60 \mathrm{~min}$. It was found to be about $130 \mathrm{~nm}$ at $t=0$ and to remain constant for about $10 \mathrm{~nm}$, achieving a plateau value of about $200 \mathrm{~nm}$ after $20 \mathrm{~min}$. This finding seems to confirm the tendency to aggregate of HSAG-SP adducts. The dependence of the particles average size on the centrifugation time was determined for the HSAG-SP-M adduct: after 5, 30, 60 and 90 min of centrifugation, $260 \mathrm{~nm}, 150 \mathrm{~nm}, 135 \mathrm{~nm}$ and $120 \mathrm{~nm}$ were respectively measured.

TEM analysis of supernatant suspensions of HSAG, HSAG-SP-T and HSAG-SP-M adducts, after 60 min of centrifugation, was performed after their deposition on a carbon film. Analysis was repeated on significant number of samples. Representative TEM micrographs are shown in Fig. 9.

HSAG in Fig. 9a is characterized by graphite stacks randomly arranged, with lateral size that appears lower than that of HSAG-SP-T (Fig. 9b) and HSAG-SP-M (Fig. 9c). This is probably due to the fact that only HSAG aggregates of small size can be

Table 3 Average size of HSAG, HSAG-SP-T and HSAG-SP-M in water dispersions, determined through DLS

\begin{tabular}{lcc}
\hline & \multicolumn{2}{c}{ Average size of particles $(\mathrm{nm})$} \\
\cline { 2 - 3 } & Starting dispersion & Supernatant dispersion \\
& & \\
HSAG & 500 & 194 \\
HSAG-SP-M & 300 & 150 \\
HSAG-SP-T & 1500 & 700 \\
${ }^{a}$ After 30 minutes centrifugation at 9000 rpm.
\end{tabular}
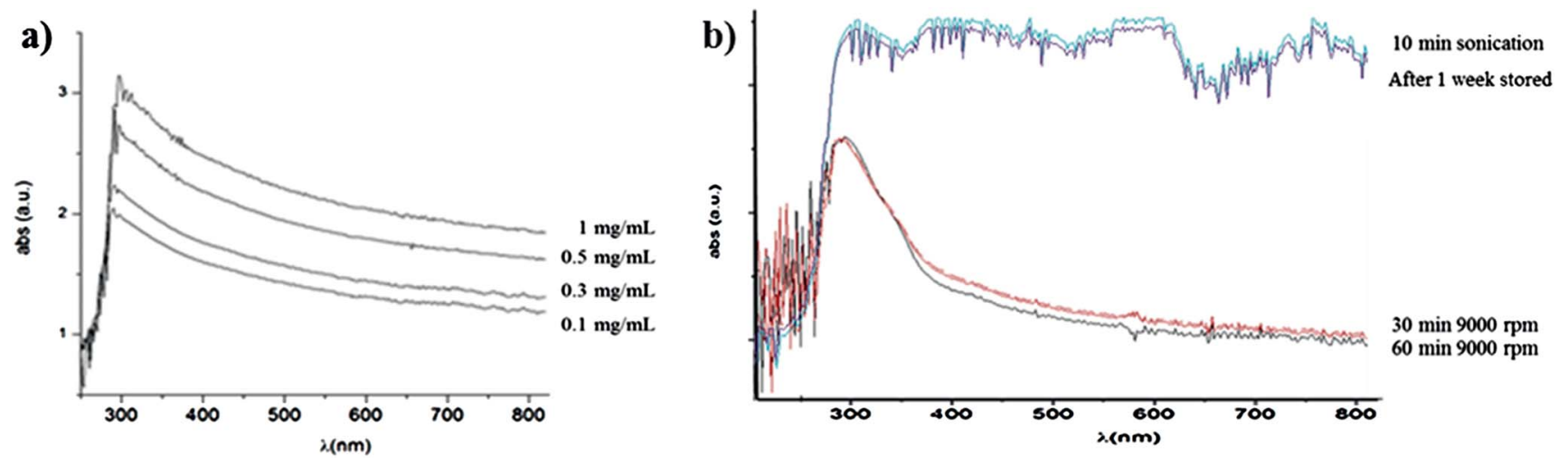

Fig. 8 Dependence of UV-vis absorbance on concentration of HSAG-SP-M adduct in water (a) and after centrifugation at 9000 rpm (b). 


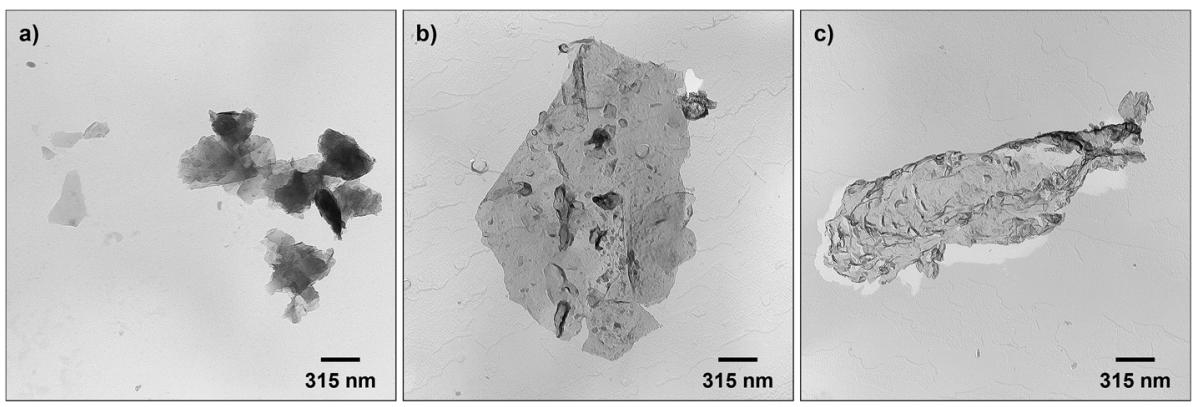

Fig. 9 TEM micrographs of water supernatant after 60 min centrifugation at 9000 rpm: HSAG (a), HSAG-SP-T (b) and HSAG-SP-M (c).

suspended in water. Micrographs of both adducts revealed stacks apparently made by a low number of layers in the case of HSAG-SP-T (Fig. 9b) and by very few layers in the case of HSAGSP-M (Fig. 9c). These findings suggest that the milling procedure promoted larger exfoliation of HSAG-SP-M adducts and, as a consequence, the formation of modified stacks of graphene layers that found it easier to remain dispersed in water, with respect to the stacks of HSAG-SP-T adducts. The difference among the average size of graphene stacks determined by DLS and the lateral size of the sheets observed in TEM micrographs seems to suggest the extreme flexibility of HSAG-SP adducts.

The structure of HSAG-SP-M adduct was investigated by performing HR-TEM analysis on samples isolated form the supernatant solutions, after centrifugation for $10 \mathrm{~min}$ at 2000 rpm and for 5 and $60 \mathrm{~min}$ at $9000 \mathrm{rpm}$. Fig. 10 shows HR-TEM micrographs at lower and higher magnifications.

Micrographs at lower magnification in Fig. 10a, c and e reveal that the lateral size of HSAG-SP-M adducts is of the same order of magnitude in samples isolated after centrifugations at different rpm and for different times. This indicates that the milling step does not cause appreciable breaking of the graphitic layers. Moreover, it appears that the SP modifier is able to bring into water solution also nano-graphites with pretty large lateral size. Micrographs at higher magnification allow to visualize the stacks of graphene layers that are disposed with a lateral side perpendicular to the beam. Thanks to this disposition of the nano-stacks, it is possible to estimate the number of stacked graphene layers. Fig. 10b shows stacks isolated after centrifugation for $10 \mathrm{~min}$ at $2000 \mathrm{rpm}$ : are visible stacks (indicated in the boxes) of about 3.5-4.2 nm, made by about 10-12 stacked graphene layers. As revealed by the exam of a good number of micrographs, such stacks are the most abundant ones in a population of stacks that contain either little larger of little lower number of layers. Fig. 10d shows a stack (indicated in the box) isolated after centrifugation for $5 \mathrm{~min}$ at $9000 \mathrm{rpm}$ : is of about $2.8 \mathrm{~nm}$ and contains about 8 stacked graphene layers. Such a stack was very frequently observed in the analyzed micrographs after centrifugation for $5 \mathrm{~min}$. In Fig. 10f it is shown the adduct isolated after centrifugation at $9000 \mathrm{rpm}$ for $60 \mathrm{~min}$. It seems that a layer of organic substance (indicated by the arrow) is tightly adhered to the carbon allotrope: voids can not be observed between the carbon surface and the organic substance. It appears that the organic substance, that has to be assumed based on $\mathbf{S P}$, is also on the graphene face. The fractionation of HSAG-SP-M adduct, as a function of centrifugation, appears to be due to the different numbers of stacked layers, rather than to different lateral size, and to the different amount of SP modifier.

It was seen in HR-TEM micrograph in Fig. 10b that the graphene nano-stacks are mostly made by about 10 graphene layers, that means quite a lower number with respect to that estimated on pristine HSAG via WAXD analysis (about 30). These findings allow to comment that reaction of HSAG with SP, through ball milling, and the successive centrifugation of water solutions of the HSAG-SP adduct lead to isolate nano-stacks

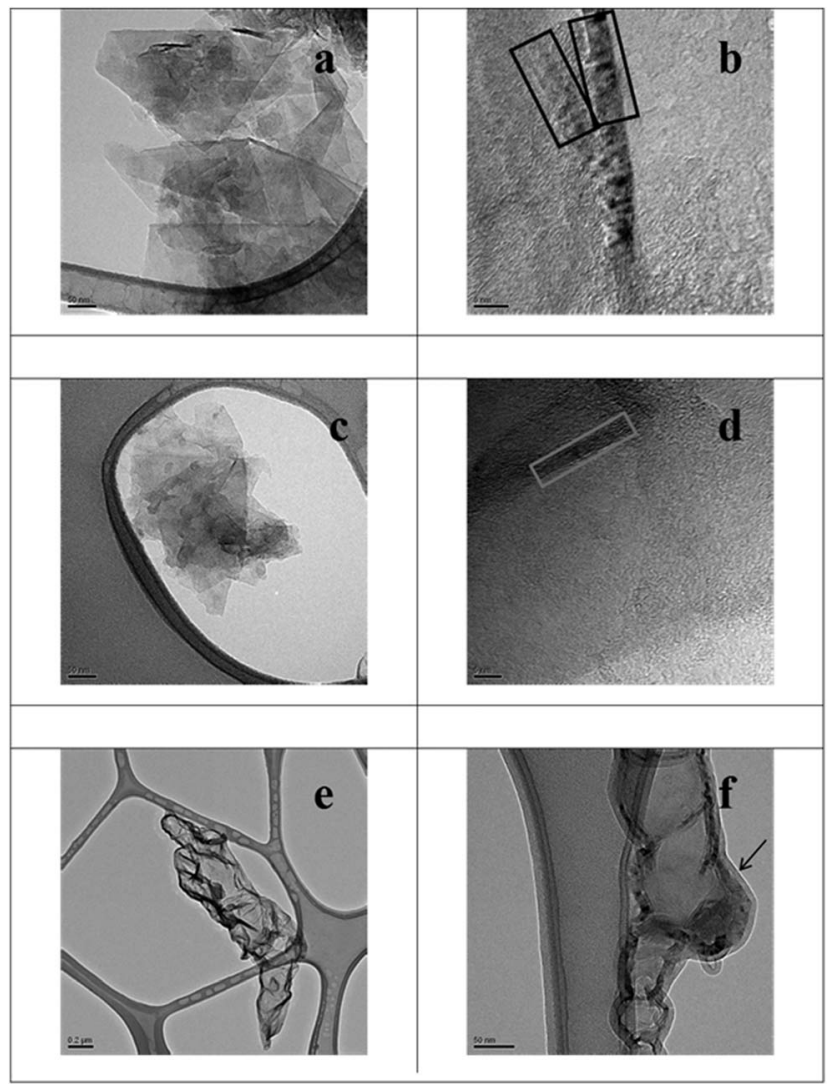

Fig. 10 Micrographs of HSAG-SP-M adducts isolated from supernatant solutions, after centrifugation for $10 \mathrm{~min}$ at $2000 \mathrm{rpm}$ (a and b), $5 \mathrm{~min}$ at $9000 \mathrm{rpm}$ (c and d) and for $60 \mathrm{~min}$ at $9000 \mathrm{rpm}$. Micrographs are: low magnification bright field TEM ( $a, c$ and e), HR-TEM images (b, d and f). 
made by few layers of graphene, whose number appears to depend on the adopted experimental conditions.

\section{Conclusions}

The neat reaction of serinol with 2,5-hexanedione led to the preparation of 2-(2,5-dimethyl-1H-pyrrol-1-yl)-1,3-propanediol, with yield of about $95 \%$ and atomic efficiency of about $78 \%$.

SP, that contains pyrrole ring, was able to form stable adducts with HSAG, a nano-graphite with high surface area and high shape anisotropy. Preparation of HSAG-SP adducts was carried out in the absence of organic solvents or catalysts, assisted by mechanical energy in a ball milling jar or by thermal energy, by simply heating the reaction flask. Adducts with $13-14 \%$ by mass of $\mathbf{S P}$ were prepared.

Functionalization reaction was successful. More than $10 \%$ by mass of non graphitic, nitrogen containing substance was detected by TGA. Functional groups, that could be attributed to SP, were revealed by IR analysis. After the reaction with SP, the same interlayer distance between graphitic layers and the same in plane order were maintained, suggesting that functionalization occurred on the edges and/or on the faces of graphite aggregates. The presence in IR spectrum of peaks other than those due to SP and HSAG leads to hypothesize that a chemical reaction occurred, with the formation of covalent bonds on the adduct, that could justify the stability of the adducts. Raman spectra of pristine HSAG and HSAG-SP adducts showed evident $\mathrm{G}$ and $\mathrm{D}$ bands. Their relative intensity was not substantially altered after the thermal reaction of HSAG with SP, suggesting that such reaction does not lead to appreciable structural modification of the graphene layers. This finding is in line with what revealed by WAXD analysis. Instead, the D band became prevailing after the ball milling treatment, that appears to play the major role in affecting the structure of the carbon allotrope.

Water solutions were prepared with HSAG-SP-M, from 0.1 to $1 \mathrm{mg} \mathrm{mL}{ }^{-1}$ as adduct concentration. Such solutions, whose UV absorbance followed the Lambert-Beer law, were stable for weeks at rest. Centrifugations were performed at different rpm and for different times, isolating HSAG-SP-M samples from the supernatant solutions. HR-TEM analysis showed that the lateral size of graphitic flakes was very similar in all the samples, whereas the number of stacked layers decreased down to at least about 8 by increasing the rpm (9000), even at short time ( $5 \mathrm{~min}$ ) of centrifugation. The HR-TEM picture of HSAG-SP-M adduct isolated at $9000 \mathrm{rpm}$ for long centrifugation time revealed that an organic layer, reasonably based on SP, was tightly adhered to the carbon surface.

The HSAG-SP adducts prepared in this work are nano-stacks of confined graphene layers, with oxygen and nitrogen containing functional groups that do not appreciably alter the solid state organization of the carbon allotrope. Thanks to such functional groups, water solutions of the graphene nanostacks can be easily formed. Moreover, centrifugation technique allow to isolate, from the supernatant solutions, HSAG-SP samples with few graphene layers.

SP is a Janus molecules and appears suitable to form stable adducts with carbon allotropes, such as HSAG, and to promote the separation of graphitic aggregates into stacks containing a low/very low number of graphene layers. Results here reported pave the way for the easy functionalization of carbon allotropes. Moreover, the easy preparation of stable dispersions in polar media allows to envisage a large variety of applications.

\section{Acknowledgements}

Dr Valeria Cipolletti (Politecnico Milano) is gratefully acknowledged for X-ray analysis and "PRIN Research Project 2010-2011" for the financial support.

\section{References}

$1 \mathrm{H}$. W. Kroto, The stability of the fullerenes $C_{n}$, with $n=24$, 28, 32, 36, 50, 60 and 70, Nature, 1987, 329(6139), 529-531.

2 S. Iijima and T. Ichihashi, Single-shell carbon nanotubes of 1 nm diameter, Nature, 1993, 363, 603.

3 D. S. Bethune, C. H. Kiang, M. S. de Vries, G. Gorman, R. Savoy, J. Vazquez, et al., Cobalt-catalysed growth of carbon nanotubes with single-atomic-layer walls, Nature, 1993, 363, 605.

4 S. Iijima, Helical microtubules of graphitic carbon, Nature, 1991, 354, 56.

5 M. Monthioux and V. L. Kuznetsov, Who should be given the credit for the discovery of carbon nanotubes?, Carbon, 2006, 44(9), $1621 \mathrm{e} 3$.

6 K. S. Novoselov, A. K. Geim, S. V. Morozov, D. Jiang, Y. Zhang, S. V. Dubonos, I. V. Grigorieva and A. A. Firsov, Electric field effect in atomically thin carbon films, Science, 2004, 306, 666-669.

7 A. K. Geim and K. S. Novoselov, The rise of graphene, Nat. Mater., 2007, 6(3), 183-191.

8 M. J. Allen, V. C. Tung and R. B. Kaner, Honeycomb carbon: a review of graphene, Chem. Rev., 2010, 110, 132-145.

9 Y. W. Zhu, et al., Graphene and graphene oxide: Synthesis, properties, and applications, Adv. Mater., 2010, 22, 39063924.

10 Y. Geng, S. J. Wang and J.-K. Kim, Preparation of graphite nanoplatelets and graphene sheets, J. Colloid Interface Sci., 2009, 336(2), 592-598.

11 L. Kavan, J. H. Yum and M. Gratzel, Optically transparent cathode for dye-sensitized solar cells based on graphene nanoplatelets, ACS Nano, 2011, 5, 165-172.

12 A. Nieto, D. Lahiri and A. Agarwal, Synthesis and properties of bulk graphene nanoplatelets consolidated by spark plasma sintering, Carbon, 2012, 50(11), 4068-4070.

13 K. R. Paton, E. Varrla, C. Backes, R. J. Smith, U. Khan, A. O'Neill, C. Boland, M. Lotya, O. M. Istrate, P. King, T. Higgins, S. Barwich, P. May, P. Puczkarski, I. Ahmed, M. Moebius, H. Pettersson, E. Long, J. Coelho, S. E. O'Brien, E. K. McGuire, B. Mendoza Sanchez, G. S. Duesberg, N. McEvoy and T. J. Pennycook, Scalable production of large quantities of defect-free few-layer graphene by shear exfoliation in liquids, Nat. Mater., 2014, 13, 624-630. 
14 J. R. Potts, D. R. Dreyer, C. W. Bielawski and R. S. Ruoff, Polymer, 2011, 52, 5.

15 C. Lee, X. Wei, J. W. Kysar and J. Hone, Measurement of the elastic properties and intrinsic strength of monolayer graphene, Science, 2008, 321(5887), 385-388.

16 A. A. Balandin, Thermal properties of graphene and nanostructured carbon materials, Nat. Mater., 2011, 10(8), 569-581.

17 Y. Zhang, Y.-W. Tan, H. L. Stormer and P. Kim, Experimental observation of the quantum hall effect and Berry's phase in graphene, Nature, 2005, 438(7065), 201-204.

18 K. K. Gomes, W. Mar, W. Ko, F. Guinea and H. C. Manoharan, Designer dirac fermions and topological phases in molecular graphene, Nature, 2012, 483(7389), 306-310.

19 M. Yi and Z. Shen, A review on mechanical exfoliation for the scalable production of graphene, J. Mater. Chem. A, 2015, 3(22), 11700-11715.

20 P. W. Sutter, J.-I. Flege and E. A. Sutter, Epitaxial graphene on ruthenium, Nat. Mater., 2008, 7(5), 406-411.

21 K. S. Kim, Y. Zhao, H. Jang, S. Y. Lee, J. M. Kim, K. S. Kim, et al., Large-scale pattern growth of graphene films for stretchable transparent electrodes, Nature, 2009, 457(7230), 706-710.

22 A. N. Obraztsov, E. A. Obraztsova, A. V. Tyurnina and A. A. Zolotukhin, Chemical vapor deposition of thin graphite films of nanometer thickness, Carbon, 2007, 45, 2017-2021.

23 A. Reina, X. Jia, J. Ho, D. Nezich, H. Son and V. Bulovic, et al., Large area, few-Layer graphene films on arbitrary substrates by chemical vapor deposition, Nano Lett., 2009, 9(1), 30-35.

24 S. J. Chae, F. Güness, K. K. Kim, E. S. Kim, G. H. Han, S. M. Kim, et al., Synthesis of largearea graphene layers on poly-nickel substrate by chemical vapor deposition: wrinkle formation, Adv. Mater., 2009, 21(22), 2328-2333.

25 X. Li, W. Cai, J. An, S. Kim, J. Nah, D. Yang, et al., Large-area synthesis of high quality and uniform graphene films on copper foils, Science, 2009, 1171245.

26 A. Reina, S. Thiele, X. Jia, S. Bhaviripudi, M. S. Dresselhaus, J. A. Schaefer, et al., Growth of large-area single- and bi-layer graphene by controlled carbon precipitation on polycrystalline Ni surfaces, Nano Res., 2009, 2, 509-516.

27 A. L. Vázquez de Parga, F. Calleja, B. Borca, M. C. G. Passeggi, J. J. Hinarejos, F. Guinea, et al., Periodically Rippled Graphene: Growth and Spatially Resolved Electronic Structure, Phys. Rev. Lett., 2008, 100, 056807.

28 Y. Hernandez, V. Nicolosi, M. Lotya, F. M. Blighe, Z. Sun, S. De and J. N. Coleman, High-yield production of graphene by liquid-phase exfoliation of graphite, Nat. Nanotechnol., 2008, 3(9), 563-568.

29 M. Lotya, et al., Liquid phase production of graphene by exfoliation of graphite in surfactant/water solutions, J. Am. Chem. Soc., 2009, 131, 3611-3620.

30 U. Khan, A. O'Neill, M. Lotya, S. De and J. N. Coleman, Highconcentration solvent exfoliation of graphene, Small, 2010, 6, 864-871.
31 U. Khan, et al., Solvent-exfoliated graphene at extremely high concentration, Langmuir, 2011, 27, 9077-9082.

32 J. N. Coleman, et al., Two-dimensional nanosheets produced by liquid exfoliation of layered materials, Science, 2011, 331, 568-571.

33 N. Nicolosi, M. Chhowalla, M. G. Kanatzidis, M. S. Strano and J. N. Coleman, Liquid exfoliation of layered materials, Science, 2013, 340, 1226419.

34 B. C. Brodie, Philos. Trans. R. Soc. London, 1859, 149, 249259.

35 L. Staudenmaier, Ber. Dtsch. Chem. Ges., 1898, 31, 14811487.

36 W. S. Hummers and R. E. Offeman, J. Am. Chem. Soc., 1958, 80, 1339.

37 D. C. Marcano, D. V. Kosynkin, J. M. Berlin, A. Sinitskii, Z. Sun, A. Slesarev, L. B. Alemany, W. Lu and J. M. Tour, Improved synthesis of graphene oxide, ACS Nano, 2010, 4(8), 4806-4814.

38 L. Sun and B. Fugetsu, Mass production of graphene oxide from expanded graphite, Mater. Lett., 2013, 109, 207-210.

39 T. Nakajima, A. Mabuchi and R. Hagiwara, A new structure model of graphite oxide, Carbon, 1988, 26(3), 357-361.

40 H. He, J. Klinowski, M. Forster and A. Lerf, A new structural model for graphite oxide, Chem. Phys. Lett., 1998, 287(1-2), 53-56.

41 Y. W. Zhu, et al., Graphene and graphene oxide: Synthesis, properties, and applications, Adv. Mater., 2010, 22, 39063924.

42 D. R. Dreyer, R. S. Ruoff and C. W. Bielawski, Angew. Chem., Int. Ed., 2010, 49, 9336-9344.

43 J. Kim, L. J. Cote and J. Huang, Two dimensional soft material: new faces of graphene oxide, Acc. Chem. Res., 2012, 45(8), 1356-1364.

44 T. Kuila, S. Bose, A. K. Mishra, P. Khanra, N. H. Kim and J. H. Lee, Chemical functionalization of graphene and its applications, Prog. Mater. Sci., 2012, 57(7), 1061-1105.

45 K. H. Liao, A. Mittal, S. Bose, C. Leighton, K. A. Mkhoyan and C. W. Macosko, Aqueous only route toward graphene from graphite oxide, ACS Nano, 2011, 5(2), 1253-1258.

46 X. G. Mei and J. Y. Ouyang, Ultrasonication-assisted ultrafast reduction of graphene oxide by zinc powder at room temperature, Carbon, 2011, 49(15), 5389-5397.

47 R. S. Dey, S. Hajra, R. K. Sahu, C. R. Raj and M. K. Panigrahi, A rapid room temperature chemical route for the synthesis of graphene: metal-mediated reduction of graphene oxide, Chem. Commun., 2012, 48(12), 1787-1789.

48 Y. M. Shul'ga, V. N. Vasilets, S. A. Baskakov, V. E. Muradyan, E. A. Skryleva and Y. N. Parkhomenko, Photoreduction of graphite oxide nanosheets with vacuum ultraviolet radiation, High Energy Chem., 2012, 46(2), 117-121.

49 A. Wei, J. Wang, Q. Long, X. Liu, X. Li, X. Dong and W. Huang, Synthesis of high-performance graphene nanosheets by thermal reduction of graphene oxide, Mater. Res. Bull., 2011, 46(11), 2131-2134.

50 S. H. Shim, K. T. Kim, J. U. Lee and W. H. Jo, Facile method to functionalize graphene oxide and its application to poly 
(ethylene terephthalate)/graphene composite, ACS Appl. Mater. Interfaces, 2012, 4(8), 4184-4191.

$51 \mathrm{~J}$. O. Besenhard, The electrochemical preparation and properties of ionic alkali metal- and NR4-graphite intercalation compounds in organic electrolytes, Carbon, 1976, 14, 111-115.

52 C. Herold, A. Herold and P. Lagrange, Ternary graphite intercalation compounds associating an alkali metal and an electronegative element or radical, Solid State Sci., 2004, 6, 125-138.

53 W. Q. Deng, X. Xu and W. A. Goddard, New alkali doped pillared carbon materials designed to achieve practical reversible hydrogen storage for transportation, Phys. Rev. Lett., 2004, 92, 166103.

$54 \mathrm{~W}$. Yan and M. M. Lerner, Synthesis and structural investigation of new graphite intercalation compounds containing the perfluoroalkylsulfonate anions $\mathrm{C}_{10} \mathrm{~F}_{21} \mathrm{SO}_{3}{ }^{-}$, $\mathrm{C}_{2} \mathrm{~F}_{5} \mathrm{OC}_{2} \mathrm{~F}_{4} \mathrm{SO}_{3}{ }^{-}$, and $\mathrm{C}_{2} \mathrm{~F}_{5}\left(\mathrm{C}_{6} \mathrm{~F}_{10}\right) \mathrm{SO}_{3}{ }^{-}$, Carbon, 2004, 42, 2981-2987.

55 J. Li, L. Vaisman, G. Marom and J. K. Kim, Br treated graphite nanoplatelets for improved electrical conductivity of polymer composites, Carbon, 2007, 45, 744-750.

56 W. Katinonkul and M. M. Lerner, Chemical synthesis of graphite bis(perfluoropinacolato)borate and graphite bis(hexafluorohydroxyisobutyrato)borate intercalation compounds, Carbon, 2007, 45, 2672-2677.

57 T. E. Sutto, T. T. Duncan and T. C. Wong, X-ray diffraction studies of electrochemical graphite intercalation compounds of ionic liquids, Electrochim. Acta, 2009, 54, 5648-5655.

58 S. Tongay, J. Hwang, D. B. Tanner, H. K. Pal, D. Maslov and A. F. Hebard, Supermetallic conductivity in bromineintercalated graphite, Phys. Rev. B: Condens. Matter Mater. Phys., 2010, 81, 115428.

59 T. Maluangnont, G. T. Bui, B. A. Huntington and M. M. Lerner, Preparation of a homologous series of graphite alkylamine intercalation compounds including an unusual parallel bilayer intercalate arrangement, Chem. Mater., 2011, 23, 1091-1095.

60 T. Quang, P. Pokharel, G. S. Song and D. S. Lee, Preparation and characterization of graphene nanoplatelets from natural graphite via intercalation and exfoliation with tetraalkylammoniumbromide, J. Nanosci. Nanotechnol., 2012, 12, 4305-4308.

61 M. Mauro, M. Maggio, V. Cipolletti, M. Galimberti, P. Longo and G. Guerra, Graphite oxide intercalation compounds with rotator hexagonal order in the intercalated Layers, Carbon, 2013, 61, 395-403.

62 M. Galimberti, V. Cipolletti, M. Mauro and L. Conzatti, Nanocomposites of Poly(1,4- cis -Isoprene) with Graphite Oxide Intercalation Compounds, Macromol. Chem. Phys., 2013, 214(17), 1931-1939.

63 S. Stankovich, D. A. Dikin, R. D. Piner, K. A. Kohlhaas, A. Kleinhammes, Y. Jia, Y. Wu, S. T. Nguyen and R. S. Ruoff, Carbon, 2007, 45, 1558-1565.
64 S. Park, J. An, J. R. Potts, A. Velamakanni, S. Murali and R. S. Ruoff, Carbon, 2011, 49, 3019-3023.

65 B. Andreeßen and A. Steinbüchel, AMB Express, 2011, 1(1), $1 \mathrm{e} 6$.

66 "Italian patent application, n. MI2014A001077", filed on June 13th, 2014, entitled "Processo per la sintesi di 2-(2,5dimetil-1-H-pirrol-1-il)-1,3-propandiolo e suoi derivati sostituiti”.

67 M. Galimberti, V. Barbera, A. Citterio, R. Sebastiano, A. Truscello, A. M. Valerio, L. Conzatti and R. Mendichi, Supramolecular interactions of carbon nanotubes with biobased polyurethanes from 2-(2, 5-dimethyl- $1 H$-pyrrol-1yl)-1, 3-propanediol, Polymer, 2015, 63, 62-70.

68 L. Knorr, Chem. Ber., 1885, 18, 299.

69 C. Paal, Chem. Ber., 1885, 18, 367.

70 "Italian patent application, n. MI2014A001497", filed on August 14th, 2014, entitled "Polimero comprendente unità ripetitive costituite da un anello pirrolico sostituito e prodotti di addizione di tali polimeri con allotropi del carbonio".

71 "Italian patent application, n. MI2014A001714", filed on October 1st, 2014, entitled "Addotti tra allotropi del carbonio e derivati del serinolo".

72 M. Mauro, V. Cipolletti, M. Galimberti, P. Longo and G. Guerra, J. Phys. Chem. C, 2012, 116(46), 24809-24813.

73 A. C. Ferrari, J. C. Meyer, V. Scardaci, C. Casiraghi, M. Lazzeri, F. Mauri and A. K. Geim, Phys. Rev. Lett., 2006, 97(18), 187401.

74 A. C. Ferrari, Solid State Commun., 2007, 143, 47.

75 S. Reich and C. Thomsen, Philos. Trans. R. Soc., A, 2004, 362, 2271.

76 M. A. Pimenta, G. Dresselhaus, M. S. Dresselhaus, L. G. Cancado, A. Jorio and R. Saito, Phys. Chem. Chem. Phys., 2007, 9, 1276.

77 C. Castiglioni, M. Tommasini and G. Zerbi, Philos. Trans. $R$. Soc., A, 2004, 362, 2425.

78 M. Tommasini, E. Di Donato, C. Castiglioni, G. Zerbi, N. Severin, T. Böhme and J. P. Rabe, AIP Conf. Proc., 2004, 723, 334, DOI: 10.1063/1.1812101.

79 D. Graf, F. Molitor, K. Ensslin, C. Stampfer, A. Jungen, C. Hierold and L. Wirtz, Nano Lett., 2007, 7, 238.

80 C. Casiraghi, A. Hartschuh, H. Qian, S. Piscanec, C. Georgi, A. Fasoli and A. C. Ferrari, Nano Lett., 2009, 9(4), 1433-1441. 81 L. R. Radovic and B. Bockrath, J. Am. Chem. Soc., 2005, 127, 5517.

82 C. Casiraghi, S. Pisana, K. S. Novoselov, A. K. Geim and A. C. Ferrari, Appl. Phys. Lett., 2007, 91(23), 233108.

83 M. J. Matthews, M. A. Pimenta, G. Dresselhaus, M. S. Dresselhaus and M. Endo, Phys. Rev. B: Condens. Matter Mater. Phys., 1999, 59, R6585.

84 M. Tommasini, C. Castiglioni, G. Zerbi, A. Barbon and M. Brustolon, Chem. Phys. Lett., 2011, 516(4), 220-224. 